\title{
A Pan-Cancer Analysis of the Prognostic Value and Expression of Adenylate Cyclase 7 (ADCY7) in Human Tumors
}

\author{
Yu Zeng $\mathbb{D}^{1, *}$ \\ Nanhong $\mathrm{Li}^{2, *}$ \\ Zhenzhen Zheng' \\ Riken Chen ${ }^{3}$ \\ Wang Liu' \\ Jinru Zhu' \\ Mingqing Zeng ${ }^{4}$ \\ Junfen Cheng' \\ Min Peng' \\ Cheng Hong ${ }^{3}$
}

'Department of Respiration, The Second Affiliated Hospital of Guangdong Medical University, Zhanjiang, Guangdong, People's Republic of China; ${ }^{2}$ Department of Pathology and Pathophysiology, Southern Medical University, Guangzhou, Guangdong, People's Republic of China; ${ }^{3}$ China State Key Laboratory of Respiratory Disease, National Clinical Research Center for Respiratory Disease, The First Affiliated Hospital of Guangzhou Medical University,

Guangzhou, Guangdong, People's Republic of China; ${ }^{4}$ First Clinical School of Medicine, Guangdong Medical University, Zhanjiang, Guangdong, People's Republic of China

*These authors contributed equally to this work
Correspondence: Cheng Hong; Min Peng Email gyfyyhc@126.com; pengminIII8@I26.com
Background: The role of adenylate cyclase 7 (ADCY7) in cancer is still unclear. This study analyzed the interrelationship between the expression and immune function of ADCY7.

Methods: ADCY7 expression in multiple human cancers was analyzed using the databases of Genotype-Tissue Expression Project (GTEx), Cancer Cell Line Encyclopedia (CCLE), and The Cancer Genome Atlas (TCGA). Correlations among ADCY7 gene expression, mismatch repair (MMR) gene expression, and DNA methyltransferase (DNMT) expression were assessed using Spearman correlation analysis. Univariate survival analysis and KaplanMeier (KM) curve were used to examine the effect of ADCY7 expression on prognosis. The Tumor Immune Estimation Resource (TIMER) database was used to evaluate the relationship between ADCY7 gene expression and tumor immune invasion or immune checkpoint gene (ICG) expression.

Results: ADCY7 was abnormally expressed in multiple human cancers and was correlated with MMR genes and DNMT expression. Univariate survival analysis and KM curve showed that ADCY7 expression influences the overall survival (OS) of six types of cancer, diseasespecific survival (DSS) of eight, and progression-free interval (PFI) of three. The high expression of ADCY7 in OS, DSS, and PFI was strongly associated with poor outcomes in patients with breast cancer and lung squamous cell carcinoma. ADCY7 expression was strongly associated with immune cell infiltration and ICG expression.

Conclusion: The results of this study indicated that ADCY7 may be a prognostic biomarker of tumorigenesis. The study may also provide a new perspective on the role of ADCY7 in human cancers.

Keywords: adenylate cyclase 7, ADCY7, prognosis, immune infiltration, pan-cancer analysis, biomarker

\section{Introduction}

Adenylate cyclase 7 (ADCY7) encodes a membrane protein that is a member of the adenylate cyclase family. It promotes transduction of extracellular signals into intracellular reactions. ${ }^{1}$ Studies have reported that ADCY7 can catalyze the generation of cyclic adenosine monophosphate (cAMP) from adenosine triphosphate (ATP). ${ }^{2,3}$ ADCY7 plays a critical role in nervous system diseases, inflammatory responses and immune responses. ${ }^{4-6}$ Studies have shown that the adenylate cyclase gene family plays a key role in tumor development. For example, ADCY2 is associated with the prognosis of malignant melanoma and colorectal cancer, ${ }^{7}$ while ADCY4 and ADCY7 are associated with poorer prognosis of acute myeloid 
leukemia. ${ }^{8}$ Among the members of the adenylate cyclase family, the function of ADCY1 in tumors have been widely investigated. ADCY1 was highly expressed and demonstrated a poorer prognosis in most cancers. 9 However, no pan-cancer analysis was performed on the association of ADCY7 with multiple tumor types.

Cancer is one of the major public problems threatening global health. This threat may be attributed to the increasing incidence of malignant tumors in patients with cancer. This increase is influenced by factors such as unhealthy lifestyle, genetic makeup, and living environment of the patients. Even in countries with very advanced medical technology, the treatment success rate for patients with cancer is very low, ${ }^{10}$ which is attributed to genetic alteration, tumor immunity, tumor recurrence, and prognosis. Thus, investigating their mechanisms was thought to improve cancer diagnosis and treatment. Given the commonality and uniqueness of tumor formation and development, analyzing the pan-cancer expression of any genes that may influence cancer occurrence and development is necessary, to evaluate the association of such genes with clinical prognosis and molecular mechanisms.

In this study, The Cancer Genome Atlas (TCGA) and other tumor research databases, such as the GenotypeTissue Expression Project (GTEx), Cancer Cell Line Encyclopedia (CCLE), cBioPortal, and Tumor IMmune Estimation Resource (TIMER), were used for the first time to access a pan-cancer analysis of ADCY7. Various factors were considered, including genetic alterations, DNA methylation, gene expression, immune infiltration, survival status, and related signaling pathways, in exploring the specific mechanisms of ADCY7 in the tumorigenesis and progression of different cancers. The results of this study revealed that ADCY7 might be significant in tumorigenesis and immune infiltration and may serve as a prognostic biomarker in many cancers.

\section{Methods}

\section{Analysis of ADCY7 Expression in Human Cancer}

The GTEx database contains the RNA-sequencing (RNAseq) data from normal tissues. ${ }^{11}$ GTEx shares its data resource on the differences in gene regulation between tissues and between individuals, which primarily involve tissue-specific gene expression and identification of the associations of gene expression levels in different tissues. In this study, the GTEx database was used to extract the
ADCY7 expression data of 31 normal tissues. In addition, the CCLE database contains information on gene expression, methylation, gene mutation, and copy number variation in cancer cells. This database was used to obtain the ADCY7 expression data of 21 tumor cell lines. TCGA database contains bioinformatics data, such as mRNA/microRNA expression profiles, copy number variation, and mutations of related cancer genes. In this study, GTEx and TCGA data were combined to perform the differential analysis of ADCY7 expression between cancer tissues and normal tissues. The expression data were normalized through $\log 2$ conversion.

\section{Genetic Alteration Analysis of ADCY7 in Human Cancer}

The cBioPortal is a cancer research database that can help in cancer tissue and cytology analysis regrading molecular data recognition and understanding of genetics, epigenetics, gene expression, and proteomics. ${ }^{12}$ This database was used to analyze genetic alteration characteristics, including missense mutation with uncharted significance, copy number amplification, deep deletion, and mRNA upregulation of ADCY7.

\section{Relationship Between ADCY7 Expression and the Expression of Mismatch Repair (MMR) Gene and DNA Methyltransferase (DNMT) Expressions}

The MMR system is a safety guarantee system used in repairing DNA base pair mismatch in human cells. ${ }^{13}$ It helps maintain the integrity and stability of the genetic material and prevents genetic mutation. TCGA database was used to extract the expressions of MMR genes, including MLH1, MSH2, MSH6, PMS2, and EPCAM, to analyze the relationship between MMR gene expression and ADCY7 expression using Spearman correlation analysis. DNA methylation, a relatively stable modification state, is an important epigenetic mechanism that can be inherited by the new offspring DNA during replication through DNMT. ${ }^{14}$ DNA methylation is a common and normal modification of eukaryotic cells and a major epigenetic form of gene expression regulation in mammals. ${ }^{15,16}$ The expressions of four DNMTs, namely, DNMT1, DNMT2, DNMT3A, and DNMT3B, were downloaded from TCGA database to perform the relationship analysis between DNMT expression and ADCY7 expression by Spearman correlation analysis. 


\section{Relationship Between ADCY7 Expression and Tumor Mutational Burden (TMB) and Microsatellite Instability (MSI) in Cancers}

TMB is associated with overall survival (OS) after immunotherapy for various cancers, suggesting that TMB can be used as a biomarker to predict the efficacy of immune checkpoint inhibitor therapy. ${ }^{17,18}$ MSI is defined as the occurrence of new microsatellite alleles at a microsatellite locus in tumors as a result of the insertion or deletion of duplicate units, compared with normal tissues. ${ }^{19}$ MSI associated with DNA MMR defects is an important clinical tumor marker. In this study, the relationship between ADCY7 expression and TMB and MSI was investigated using Spearman correlation analysis.

\section{Immune Correlation Analysis}

The TIMER database is a free and publicly accessible database where immune cell infiltration in tumor tissues is detected using RNA-seq data. ${ }^{20}$ Tumor-infiltrating immune cells are critical to the spread of cancer. In rumors macrophages and monocytes may serve as specific biomarkers for cancer, helping physicians develop novel cancer diagnostic and therapeutic strategies. In this study, the TIMER database was used to investigate the relationship between ADCY7 expression and immune infiltrates based on all TCGA cancers. The relationship between ADCY7 expression and six types of immune cells was evaluated using Spearman correlation analysis.

\section{Survival Prognosis Analysis}

TCGA database also contains clinical data on a variety of human cancers, including information on the patients' prognosis. This information, including OS time, diseasespecific survival (DSS) time, and progression-free interval (PFI) time, was downloaded from TCGA database. The relationship between prognosis and ADCY7 expression was investigated in 33 types of cancer. The forest plots and Kaplan-Meier (KM) curves were used to present the results.

\section{ADCY7-Correlated Gene Functional Enrichment Analyses}

The STRING database is another free and publicly accessible database that can be used to explore protein-protein interactions. ${ }^{21}$ First, the top 50 genes closely correlated with ADCY7 were screened by the STRING database. The thresholds set were set as follows: the organism was Homo sapiens, the minimum interaction score was 0.9 , the meaning of network edges was evidence, the maximum number of interactors to show was less than 50. Moreover, ADCY7 and its correlated genes were enriched and analyzed using the "clusterProfiler" package in R software for Gene Ontology (GO) and Kyoto Encyclopedia of Genes and Genomes (KEGG) pathways, respectively. ${ }^{22,23}$

\section{Statistical Analysis}

Kruskal-Wallis test was used to analyze ADCY7 expression levels in different normal tissues and different tumor cell lines. Differences in ADCY7 expression levels in tumor tissues and normal tissues were evaluated by $t$-test. Univariate survival analysis was used to analyze the correlation of ADCY7 expression and patients' survival. KM methods were employed to compare survival by different levels of BRAP expression. Spearman correlation analysis was performed between BRAP expression and MMR gene mutation level, methyltransferases level, and immune checkpoint marker level. Correlations were considered significant and positive at $\mathrm{P}<0.05$ and $\mathrm{r}>0.20 . \mathrm{P}<0.05$ was considered significant for all statistical analyses.

\section{Results \\ Pan-Cancer Expression Analysis of ADCY7}

First, ADCY7 expression in 31 human tissues was analyzed using the GTEx database. Figure 1A shows that ADCY7 had high expressions in the bone marrow, lung, ovary, and spleen, but low expressions in the muscle, pancreas, and kidney tissues. Then, ADCY7 expressions in 21 human tumor cells were analyzed using the CCLE database. As shown in Figure 1B, ADCY7 was expressed in all 21 tumor cells. Moreover, the differential expression analysis of ADCY7 between normal tissues and 20 types of tumors was performed using TCGA database. The mRNA expression of ADCY7 was higher in bladder cancer (BLCA), cholangiocarcinoma (CHOL), esophageal carcinoma (ESCA), head and neck squamous cell carcinoma (HNSC), kidney renal clear cell carcinoma (KIRC), kidney renal papillary cell carcinoma (KIRP), liver hepatocellular carcinoma (LIHC), stomach adenocarcinoma (STAD), and uterine corpus endometrial carcinoma (UCEC) tissues than in normal tissues but lower in lung adenocarcinoma (LUAD) and lung squamous cell carcinoma (LUSC) tissues than in normal tissues (Figure 1C). Since few normal tissues were included in TCGA 
Kruskal-Wallis test $p=0$

A

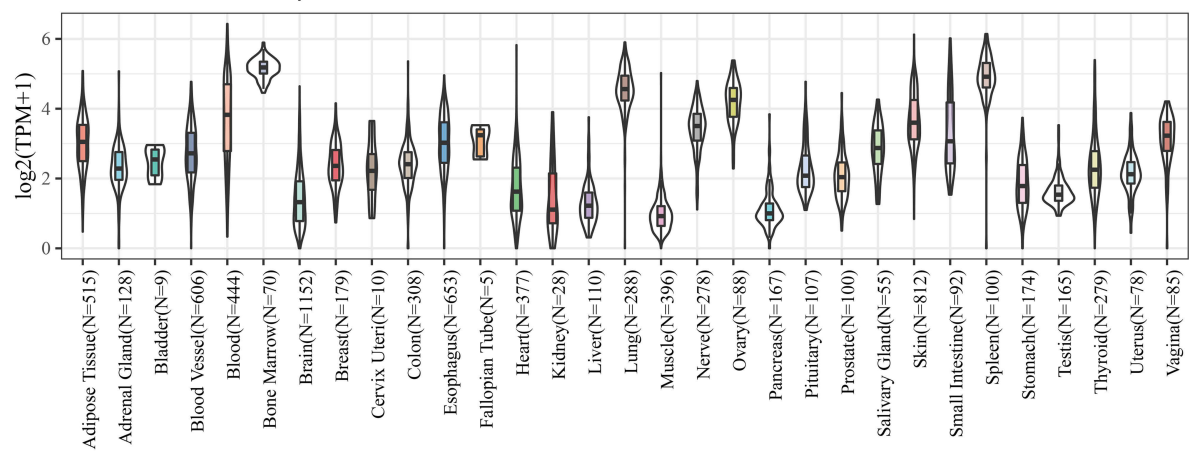

B
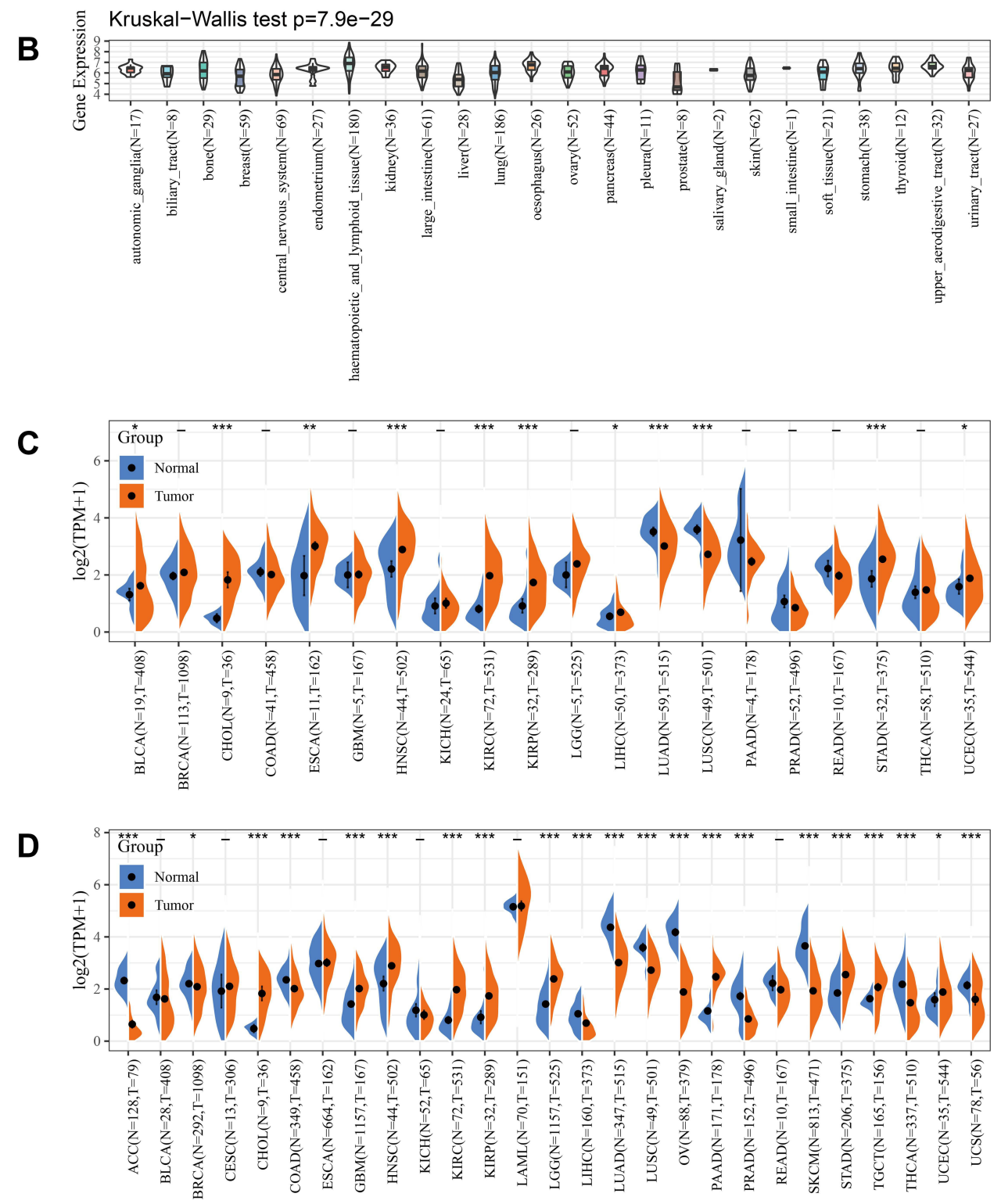

Figure I The expression of ADCY7 in cancers. (A) The expression of ADCY7 in 31 tissues (GTEx). (B) The expression of ADCY7 in twenty-one kinds of tumor cells (CCLE). (C) Differential expression of ADCY7 in cancers and normal tissues from TCGA database. (D) ADCY7 is differentially expressed in 22 tumor tissues from the GTEx database and TCGA database. *P-value < 0.05, **P-value < 0.0I, ***P-value < $0.00 \mathrm{I}$, - No significant.

Abbreviations: ADCY7, Adenylate cyclase 7; GTEx, Genotype-Tissue Expression Project; CCLE, Cancer Cell Line Encyclopedia; TCGA, The Cancer Genome Atlas. 
database, TCGA and GTEx databases were merged for differential expression analysis. As shown in Figure 1D, ADCY7 was differentially expressed in 22 tumor tissues, including up-expression in CHOL, glioblastoma multiforme (GBM), HNSC, KIRC, KIRP, brain lower-grade glioma (LGG), pancreatic adenocarcinoma (PAAD), STAD, testicular germ cell tumors (TGCT), and UCEC tumor tissues, and down-expression in adenoid cystic carcinoma (ACC), breast cancer (BRCA), colon adenocarcinoma (COAD), LUAD, lung squamous cell carcinoma (LUSC), LIHC, thyroid carcinoma (THCA), ovarian serous cystadenocarcinoma (OV), uterine carcinosarcoma (UCS), prostate adenocarcinoma (PRAD), and skin cutaneous melanoma (SKCM) tumor tissues compared with normal tissues. ADCY7 is abnormally expressed in numerous tumors.

\section{Genetic Alteration Analysis}

A genomic mutation is the main driving factor in the occurrence and development of tumors. The cBioportal database was used to analyze the characteristics of ADCY7 genetic alterations (Figure 2). ADCY7 mutation is one of the most important factors because of its expression alteration in the following tumor tissues: SKCM, UCEC, stomach adenocarcinoma, LUAD, colorectal adenocarcinoma, bladder urothelial carcinoma, LUSC, esophageal adenocarcinoma, glioblastoma multiforme, HNSCC, adrenocortical carcinoma, CSCC, thymoma, KIRC, KIRP, brain lower grade glioma, and thyroid carcinoma. The deep deletion of ADCY7 is one of the most important factors due to its expression alteration in the following tumor tissues: uterine carcinosarcoma, diffuse large B-cell lymphoma, sarcoma, prostate adenocarcinoma, breast-invasive carcinoma, mesothelioma, and liver hepatocellular carcinoma.

\section{Relationship Between ADCY7 Expression, MMR Genes, and DNA Methyltransferase Expression}

To study the role of ADCY7 in the genesis and development of tumors, the relationship between ADCY7 expression, MMR genes, and DNMTs expression were analyzed. The expression of ADCY7 was associated with the

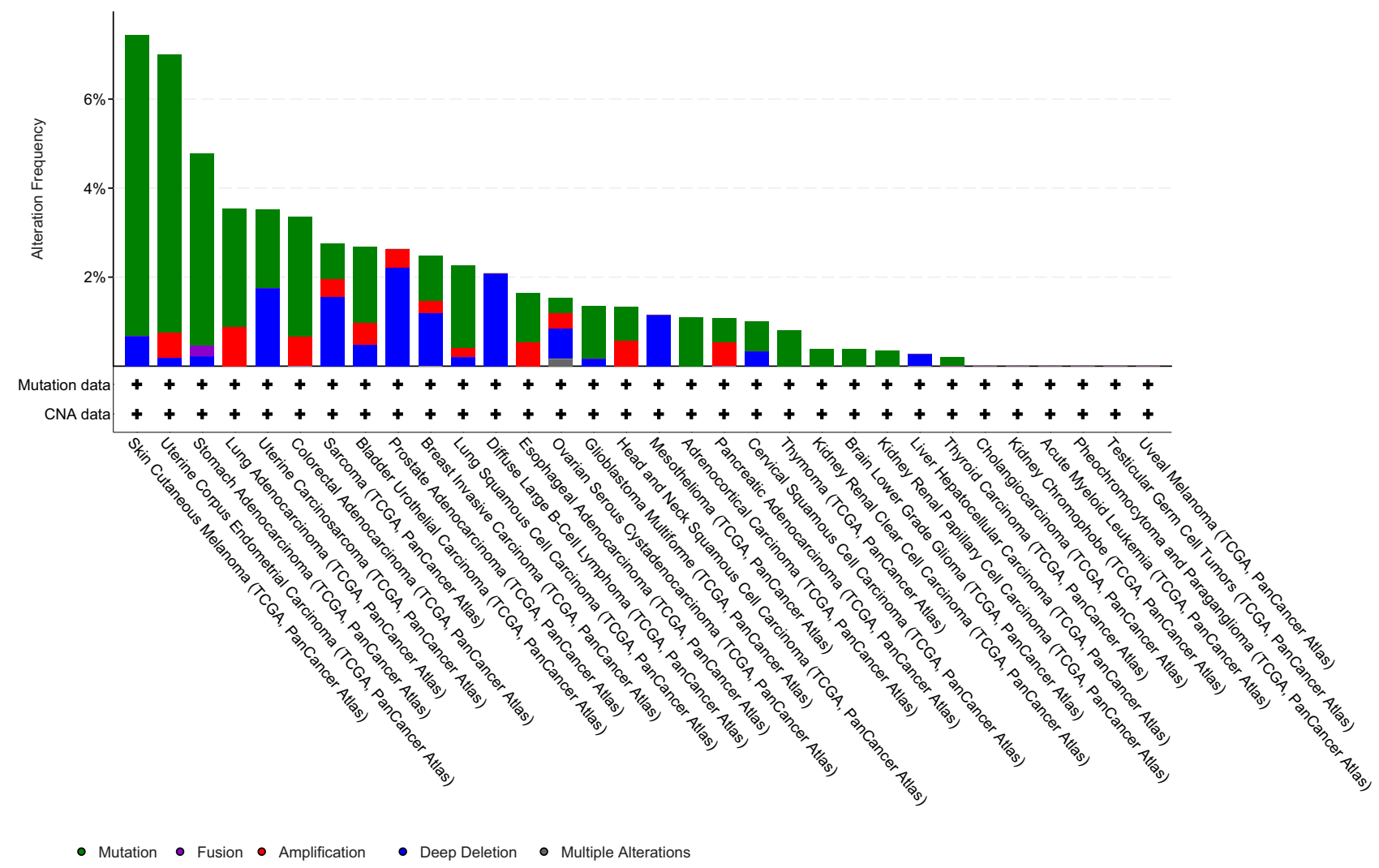

Figure 2 The kinds of genetic alterations and frequency of ADCY7 in different tumors (cBioPortal). The cBioPortal database was applied to study the ADCY7 mutation in cancers. The results are displayed as a histogram of the alteration frequencies of ADCY7 across cancer studies. Color images are available online. Abbreviation: ADCY7, Adenylate cyclase 7. 
expression of five MMR genes in most tumors, except for ACC and GBM (Figure 3A). The connection analysis between ADCY7 expression and 4 DNMTs expression was performed. Figure $3 \mathrm{~B}$ shows a strong relationship between ADCY7 expression and expressions of four DNMTs in cancers, except for ESCA, GBM, and ACC. The result suggested that ADCY7 may regulate the DNA damage process and repair of the methylation status of related genes so that they can participate in the development of human cancers.

\section{Relationship Between ADCY7 Expression and $T M B$ and $M S I$ in Cancers}

In the study, the relationship between ADCY7 expression and TMB and MSI were analyzed. Figure 4A shows that ADCY7 expression had positive relevance to TMB in $\mathrm{BRCA}, \mathrm{COAD}$, and $\mathrm{UCEC}$, and negative relevance to TMB in LIHC and THCA. In addition, ADCY7 expression was positively related to MSI in CESC, COAD, and LUSC, and negatively related to DLBC, HNSC, and SKCM (Figure 4B). These results indicated that ADCY7 expression was related to immunity in different types of cancer.

\section{Immune Correlation Analysis}

The immune cells' infiltration of tumor tissue is closely in matter of clinical outcomes and can act as therapeutic targets to improve the survival time. Therefore, the study explored the dependency of the immune infiltration level and ADCY7 expression level in 33 cancers using the TIMER database. Results show that ADCY7 expression was related to the infiltration of six types of immune cells in ACC, BRCA, and CHOL (Figure 5).

\section{Survival Prognosis Analysis}

The study also explored how ADCY7 expression affected the prognosis (OS, DSS, DFI, and PFI) of patients with cancer. According to the univariate survival analysis of 33 types of cancer, the results revealed that ADCY7 expression was correlated with OS in patients with BLCA, LUSC, MESO, OV, PCPG, and SKCM (Figure 6A). KM curves presented that the overexpression of ADCY7 indicated poorer prognosis in $\mathrm{BLCA}(\mathrm{P}<0.0001, \mathrm{HR}=1.07)$, LUSC $\quad(\mathrm{P}=0.004, \quad \mathrm{HR}=1.04), \quad \mathrm{MESO} \quad(\mathrm{P}<0.0001$, $\mathrm{HR}=1.07), \quad \mathrm{OV} \quad(\mathrm{P}=0.0073, \quad \mathrm{HR}=1.06), \quad$ and $\mathrm{PCPG}$ $(\mathrm{P}=0.0035, \mathrm{HR}=1.93)$, but better prognosis in $\mathrm{SKCM}$ $(\mathrm{P}=0.0018, \mathrm{HR}=0.96)$ (Figure $6 \mathrm{~B})$. The study also found that ADCY7 expression was associated with patients' DSS in eight types of cancer, seven of which were associated with worse prognosis, including BLCA $(\mathrm{P}<0.0001$, $\mathrm{HR}=1.07), \quad \mathrm{COAD} \quad(\mathrm{P}=0.0071, \quad \mathrm{HR}=1.09), \quad$ LUSC $(\mathrm{P}=0.00032, \mathrm{HR}=1.06)$, MESO $(\mathrm{P}=0.0004, \mathrm{HR}=1.07)$, PCPG $\quad(\mathrm{P}=0.0012, \quad \mathrm{HR}=2.38), \quad \mathrm{STAD} \quad(\mathrm{P}=0.017$, $\mathrm{HR}=1.03)$, and UVM $(\mathrm{P}=0.0011, \mathrm{HR}=1.49)$, while one was related to better prognosis in SKCM $(\mathrm{P}=0.0078$, $\mathrm{HR}=0.95) \quad$ (Figure 7A and $\mathrm{B}$ ). Regarding PFI, results revealed that $\mathrm{ADCY} 7$ expression was associated with patients' $\mathrm{PFI}$ in three types of cancer, including BLCA, LUSC, and UVM (Figure 8A). KM curves showed that the overexpression of ADCY7 was associated with better prognosis in BLCA $(\mathrm{P}<0.0001, \mathrm{HR}=1.07)$, LUSC $(\mathrm{P}<0.0001, \mathrm{HR}=1.05)$, and UVM $(\mathrm{P}=0.024, \mathrm{HR}=1.81)$ (Figure $8 \mathrm{~A}$ and $\mathrm{B}$ ).

\section{ADCY7 and Its Correlated Gene Functional Enrichment Analyses}

To investigate the molecular mechanism of ADCY7 in the occurrence and development of diseases, ADCY7 and its correlated genes were enriched and analyzed by the "clusterProfiler" package in R software (version 3.6.3) for GO and KEGG pathways, respectively. In GO terms, ADCY7 and its correlated gene were mainly enriched in the following: cAMP-mediated signaling, cyclicnucleotide-mediated signaling, second-messengermediated signaling, cAMP-dependent protein kinase complex, heterotrimeric G-protein complex, GTPase complex, ciliary base, catecholamine binding, G-protein beta/gamma-subunit complex binding, peptide binding, and dopamine neurotransmitter receptor activity (Figure 9). In KEGG terms, ADCY7 and its correlated gene were mainly enriched in the following: neuroactive ligand-receptor interaction, cAMP signaling pathway, dopaminergic synapse, morphine addiction, renin secretion, circadian entrainment, regulation of lipolysis in adipocytes, glutamatergic synapse, gap junction, and serotonergic synapse (Figure 10).

\section{Discussion}

cAMP serves as a second messenger in the immune system. It regulates cell proliferation, differentiation, and apoptosis. $^{24,25}$ Adenylate cyclase regulates intracellular cAMP concentration. Studies have shown that ADCY7 can catalyze the generation of cAMP from ATP. ${ }^{26,27}$ Therefore, ADCY7 may be involved in cell proliferation, 
A

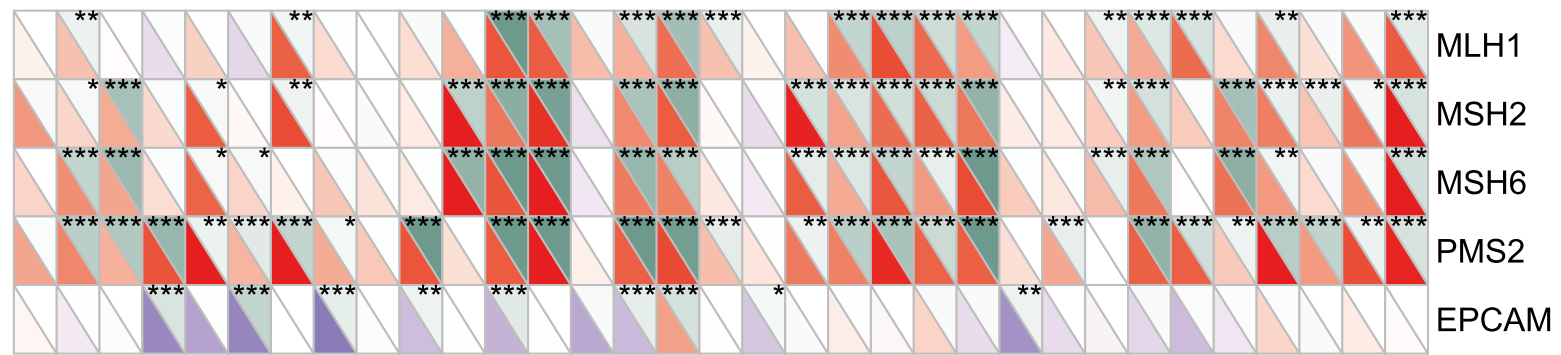

论过

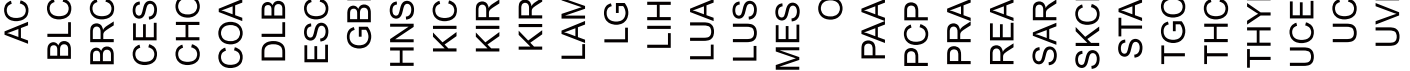

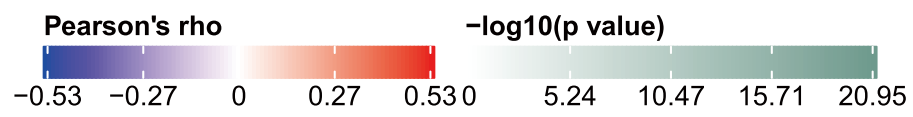

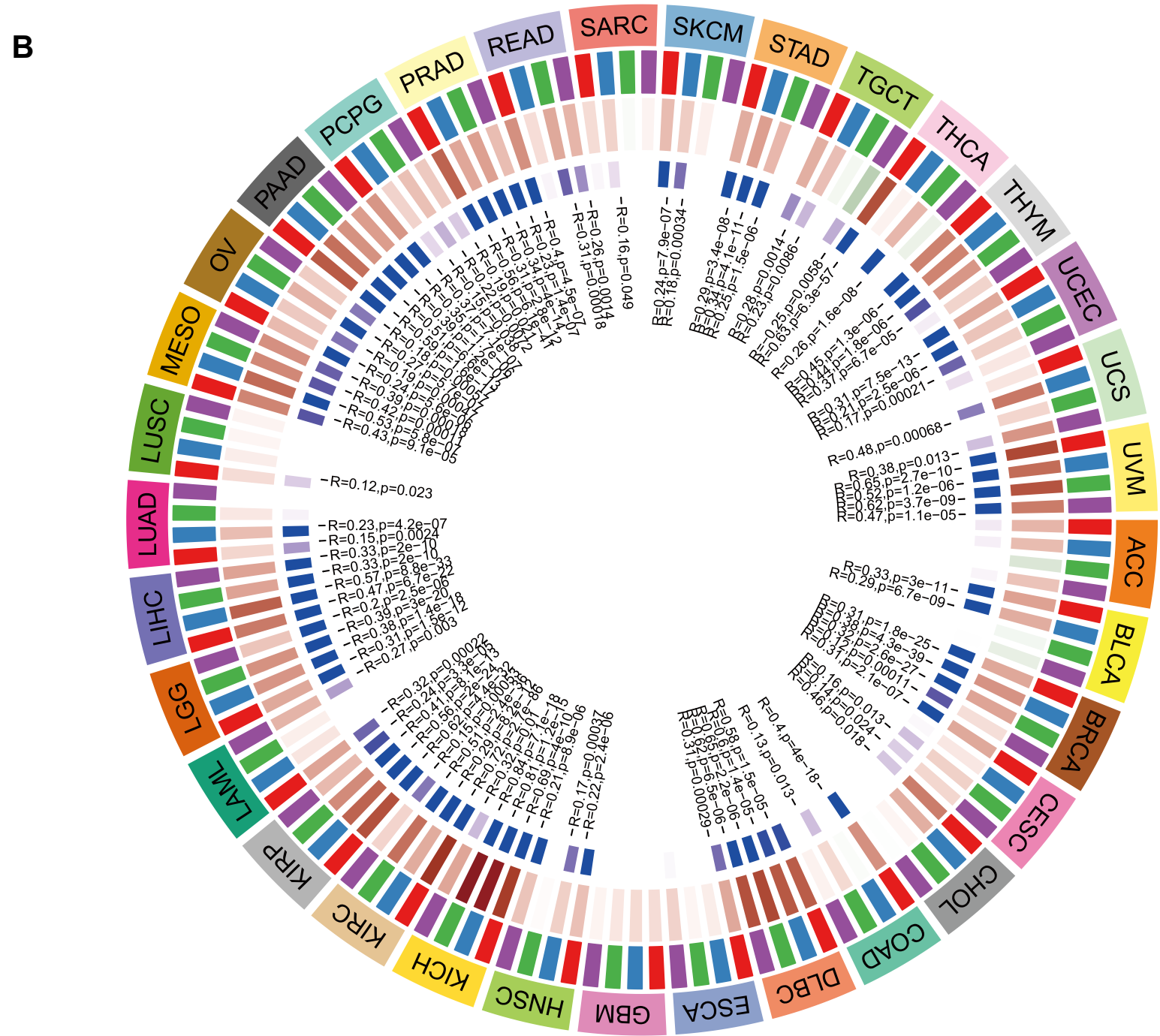

Figure 3 The expression of ADCY7 is related to the expression of five MMR genes and DNMTs expression in cancers. (A) The relationship between the expression of $A D C Y 7$ and expression of MMR genes in cancers. The expression of ADCY7 was associated with the expression of five MMR genes in most tumors, except for ACC and GBM. (B) The relationship between the expression of ADCY7 and expression of DNMTs in cancers. Strong relationship between ADCY7 expression and expressions of four DNMTs in cancers, except for ESCA, GBM, and ACC. *P-value $<0.05$, **P-value $<0.01$, ***P-value $<0.001$.

Abbreviations: ADCY7, Adenylate cyclase 7; MMR, Mismatch repair; DNMTs, DNA methyltransferases. 
A

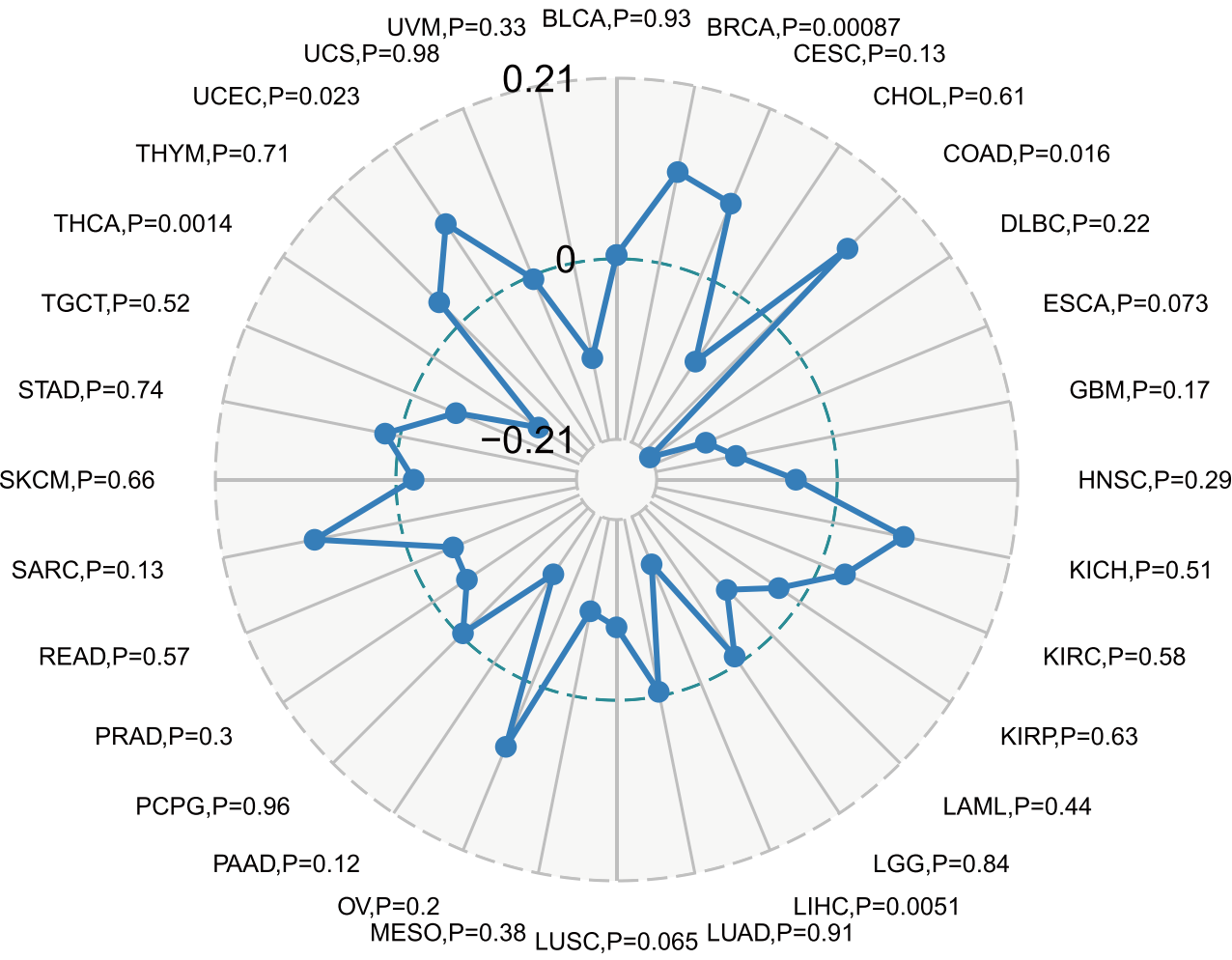

B

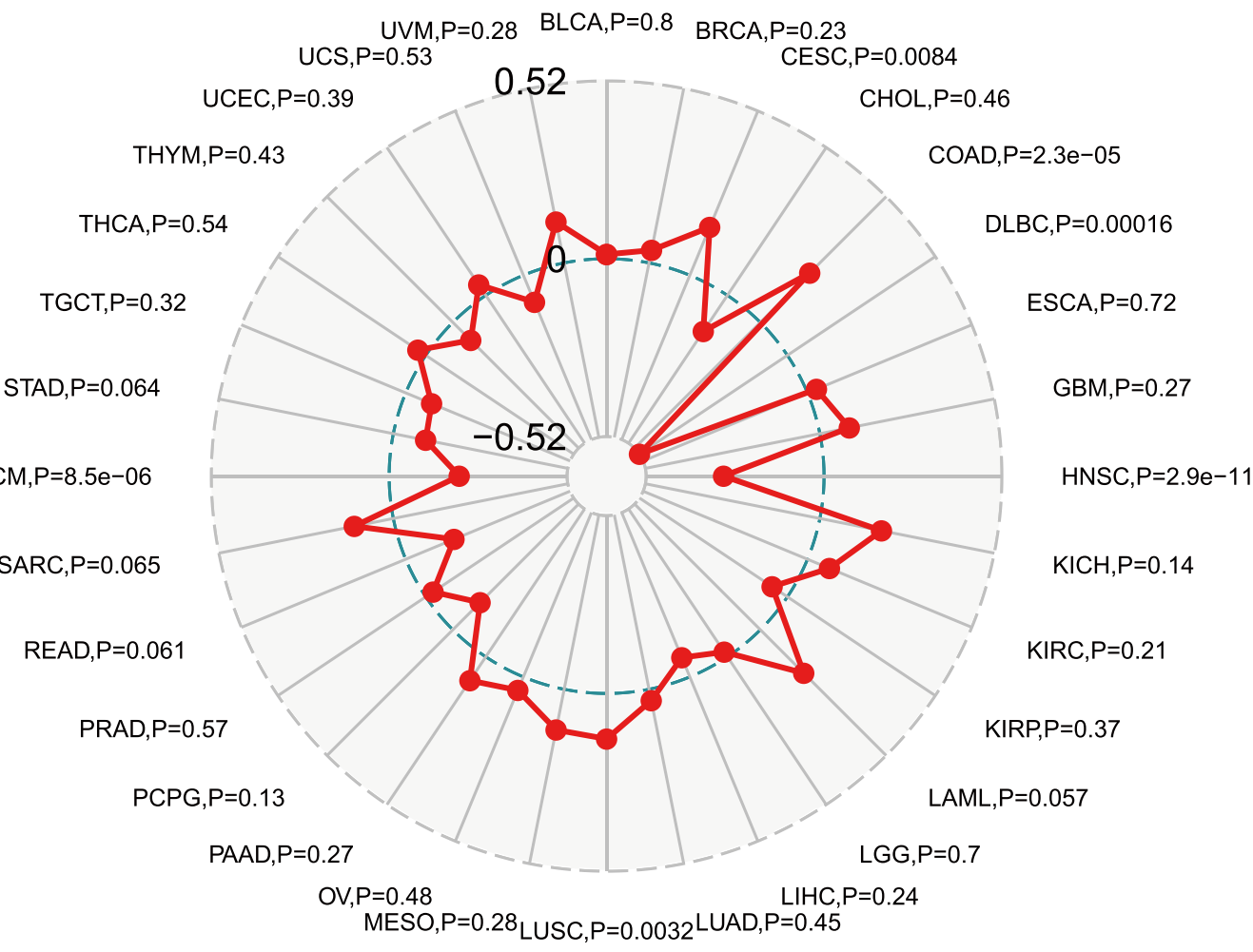

Figure 4 Correlation between ADCY7 expression and TMB and MSI in human cancers. (A) Radar map of correlation between ADCY7 expression and TMB. ADCY7 expression had positive relevance to TMB in BRCA, COAD, and UCEC, and negative relevance to TMB in LIHC and THCA. (B) Radar map of correlation between ADCY7 expression and MSI. ADCY7 expression was positively related to MSI in CESC, COAD, and LUSC, and negatively related to DLBC, HNSC, and SKCM.

Abbreviations: ADCY7, Adenylate cyclase 7; TMB, Tumor mutational burden; MSI, Microsatellite instability. 


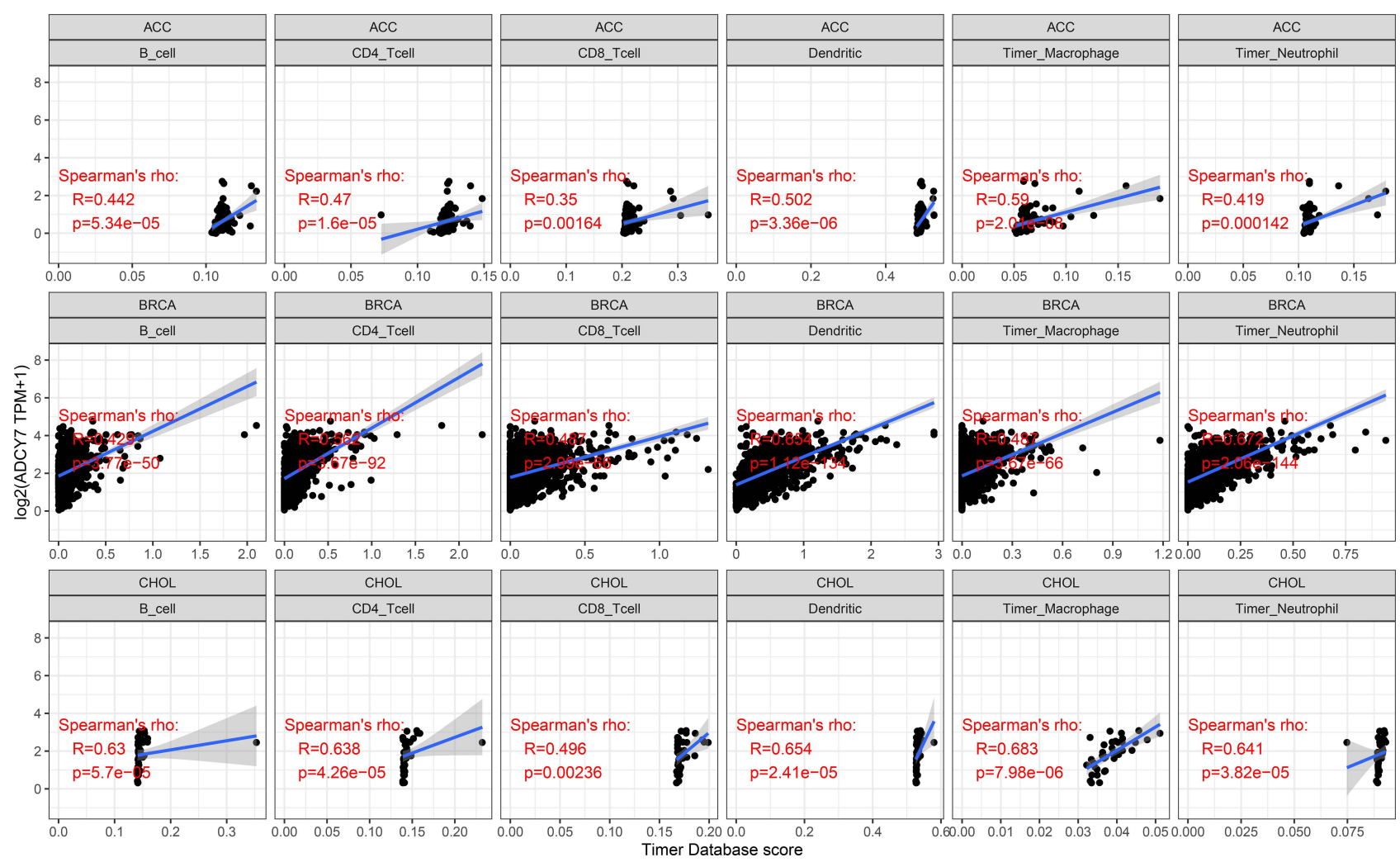

Figure 5 Correlation of $A D C Y 7$ expression with immune infiltration level in ACC, BRCA, and CHOL. ADCY7 expression is positively correlated with immune infiltration in $\mathrm{ACC}, \mathrm{BRCA}$, and $\mathrm{CHOL}$.

Abbreviation: ADCY7, Adenylate cyclase 7.

differentiation, and apoptosis by regulating cAMP. It has been reported that targeting cAMP signaling pathway can be used in the treatment of chronic lymphocytic leukemia. ${ }^{28}$ Cancer has many common biological characteristics, such as abnormal cell proliferation and differentiation, invasion, and metastasis. Thus, it can be hypothesized that ADCY7 may be involved in the genesis and progression of cancer. Although the role of ADCY7 in acute myeloid leukemia has been demonstrated, its role in pervasive cancer and its potential to be used as a biomarker are unclear. ${ }^{29}$ In this study, TCGA and other tumor research databases (including GTEx, CCLE, cBioportal, and TIMER) were used in the pan-cancer analysis of ADCY7. According to the study's results, it was found that $\mathrm{ADCY} 7$ was expressed differently in numerous cancers, and the number of ADCY7 alternations was high in most cancer types. According to the Cox and KM survival analyses, abnormal expression of ADCY7 may be a prognostic factor for some types of cancer. The study also predicted that ADCY7 dysregulation was related to cancer immunity, MMR, and DNA methylation. Therefore, ADCY7 plays a significant role in tumor immunity and might serve as a potential biomarker for prognosis.

Precision medicine has allowed us to identify specific tumor targets or characteristics for individualized treatment and cure. ${ }^{30}$ The pan-cancer analysis of ADCY7 is significant in investigating differential expressions of ADCY7 and their relative function in cancers. ${ }^{31}$ Abnormal gene expression is closely related to tumorigenesis and invasion. To the best of our knowledge, this is the first study to find abnormal expressions of ADCY7 in human cancers, including CHOL, GBM, HNSC, KIRC, KIRP, LGG, PAAD, STAD, TGCT, UCEC, ACC, BRCA, COAD, LIHC, LUAD, LUSC, OV, PRAD, SKCM, THCA, and UCS. This suggests that ADCY7 may be related to the occurrence and progression of these cancers.

MMR is closely related to MSI, and the loss of MMR expression leads to the numerous errors in gene replication. This will further affect the MSI and change the DNA repeat sequence, leading to the occurrence of tumors. ${ }^{32}$ Defective DNA mismatch repair (dMMR) and MSI high (MSI-H) are important indicators of the effectiveness of immune checkpoint inhibitors. ${ }^{33}$ For patients with 


\begin{tabular}{|c|c|c|}
\hline & HR & $P$ Value \\
\hline ACC & $1.01(0.71 \sim 1.44)$ & 0.95000 \\
\hline BLCA & $1.07(1.03 \sim 1.1)$ & 0.00034 \\
\hline BRCA & $1.02(0.97 \sim 1.07)$ & 0.44000 \\
\hline CESC & $1.04(0.99 \sim 1.09)$ & 0.11000 \\
\hline $\mathrm{CHOL}$ & $0.87(0.68 \sim 1.1)$ & 0.23000 \\
\hline COAD & $1.05(0.99 \sim 1.12)$ & 0.10000 \\
\hline DLBC & $1.01(0.92 \sim 1.1)$ & 0.91000 \\
\hline ESCA & $0.98(0.93 \sim 1.02)$ & 0.27000 \\
\hline GBM & $1.01(0.95 \sim 1.07)$ & 0.82000 \\
\hline HNSC & $1(0.98 \sim 1.02)$ & 0.94000 \\
\hline $\mathrm{KICH}$ & $1.06(0.53 \sim 2.11)$ & 0.86000 \\
\hline KIRC & $1.01(0.96 \sim 1.06)$ & 0.67000 \\
\hline KIRP & $1.08(0.98 \sim 1.18)$ & 0.11000 \\
\hline LAML & $1.01(1 \sim 1.01)$ & 0.05900 \\
\hline LGG & $1.05(1 \sim 1.1)$ & 0.07200 \\
\hline LIHC & $1.12(0.99 \sim 1.26)$ & 0.06200 \\
\hline LUAD & $0.99(0.97 \sim 1.01)$ & 0.26000 \\
\hline LUSC & $1.04(1.01 \sim 1.08)$ & 0.01300 \\
\hline MESO & $1.07(1.02 \sim 1.12)$ & 0.00320 \\
\hline OV & $1.06(1.01 \sim 1.11)$ & 0.02300 \\
\hline PAAD & $1.01(0.97 \sim 1.05)$ & 0.68000 \\
\hline PCPG & $1.93(1.22 \sim 3.05)$ & 0.00520 \\
\hline PRAD & $0.94(0.45 \sim 1.98)$ & 0.87000 \\
\hline READ & $0.85(0.69 \sim 1.06)$ & 0.15000 \\
\hline SARC & $0.98(0.93 \sim 1.04)$ & 0.48000 \\
\hline SKCM & $0.96(0.92 \sim 1)$ & 0.03100 \\
\hline STAD & $1.02(0.99 \sim 1.04)$ & 0.28000 \\
\hline TGCT & $1.23(0.88 \sim 1.71)$ & 0.23000 \\
\hline THCA & $1.06(0.87 \sim 1.29)$ & 0.57000 \\
\hline THYM & $0.89(0.7 \sim 1.13)$ & 0.33000 \\
\hline UCEC & $0.97(0.91 \sim 1.04)$ & 0.35000 \\
\hline UCS & $0.98(0.83 \sim 1.16)$ & 0.84000 \\
\hline UVM & $1.4(0.97 \sim 2.02)$ & 0.07400 \\
\hline
\end{tabular}

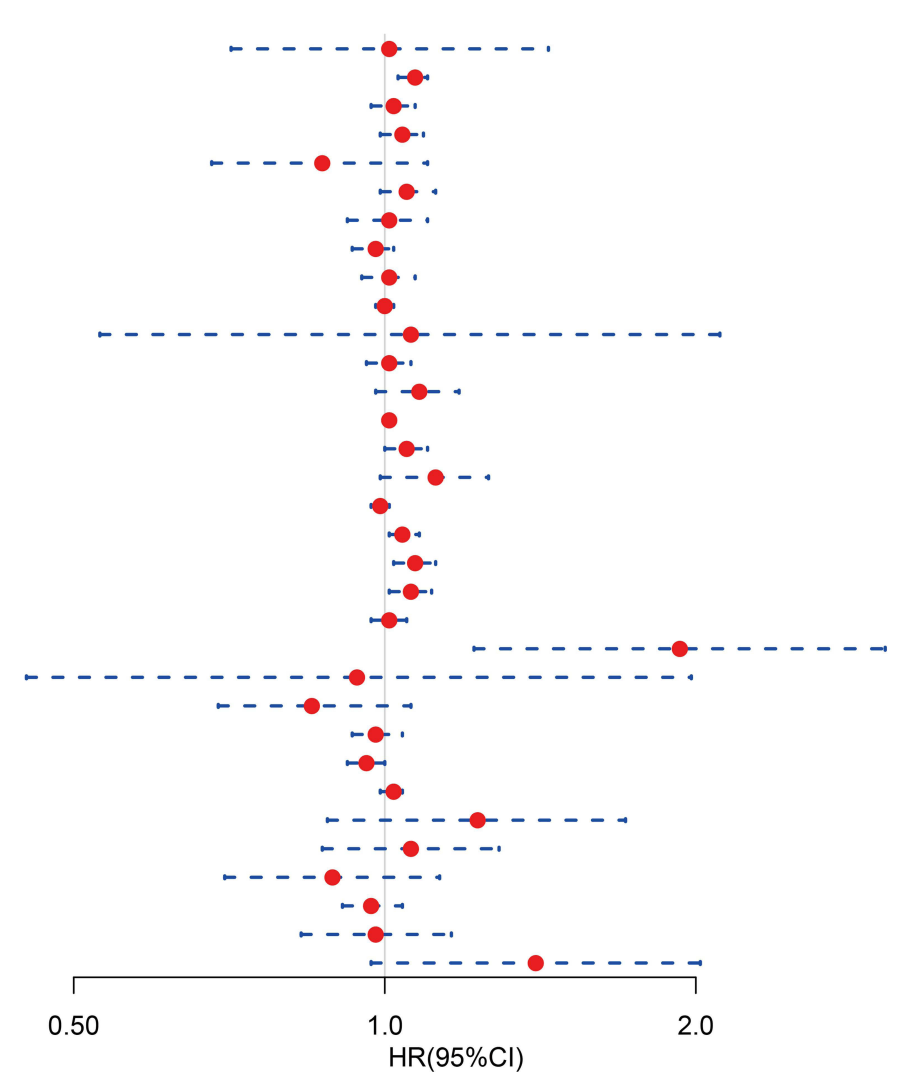

B
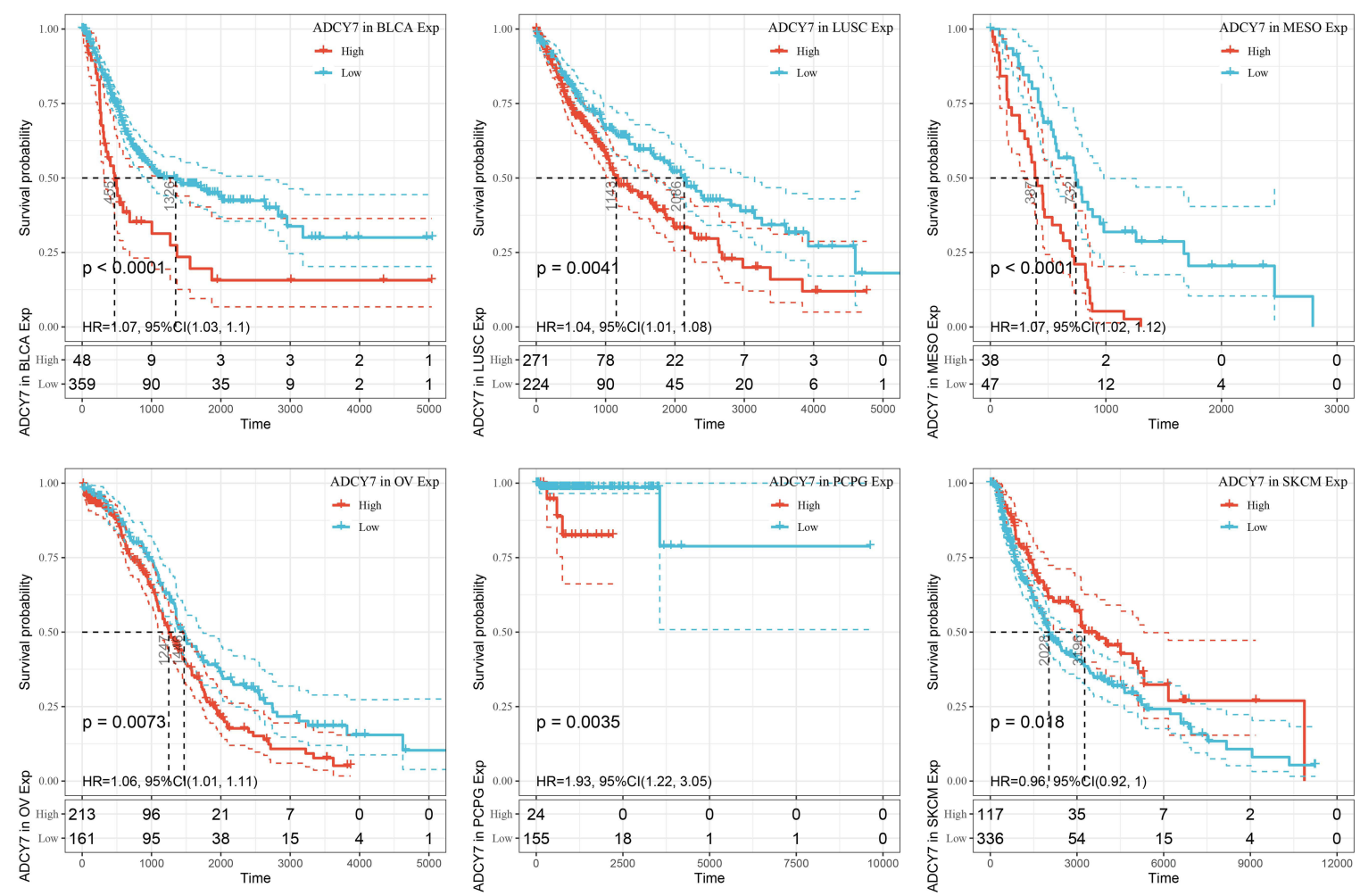

Figure 6 The relationship between ADCY7 expression and OS. (A) Forest plots of ADCY7 in thirty-three kinds of cancers. (B) KM curves showed that ADCY7 expression was correlated with OS in 6 kinds of cancers (BLCA, LUSC, MESO, OV, PCPG, and SKCM).

Abbreviations: ADCY7, Adenylate cyclase 7; OS, Overall survival. 
A

$\begin{array}{lrr} & \text { HR } & \text { P Value } \\ \text { ACC } & 1.01(0.7 \sim 1.46) & 0.94000 \\ \text { BLCA } & 1.07(1.03 \sim 1.12) & 0.00054 \\ \text { BRCA } & 1.03(0.97 \sim 1.1) & 0.32000 \\ \text { CESC } & 1.03(0.97 \sim 1.09) & 0.34000 \\ \text { CHOL } & 0.88(0.69 \sim 1.13) & 0.32000 \\ \text { COAD } & 1.09(1.02 \sim 1.17) & 0.00990 \\ \text { DLBC } & 1.03(0.9 \sim 1.17) & 0.66000 \\ \text { ESCA } & 0.99(0.94 \sim 1.04) & 0.57000 \\ \text { GBM } & 1.02(0.96 \sim 1.08) & 0.57000 \\ \text { HNSC } & 1(0.97 \sim 1.03) & 0.81000 \\ \text { KICH } & 1.25(0.63 \sim 2.46) & 0.52000 \\ \text { KIRC } & 1.01(0.95 \sim 1.07) & 0.68000 \\ \text { KIRP } & 1.09(0.98 \sim 1.21) & 0.09800 \\ \text { LAML } & \text { NA(NA N NA) } & \\ \text { LGG } & 1.05(1 \sim 1.11) & 0.05300 \\ \text { LIHC } & 1.09(0.93 \sim 1.29) & 0.29000 \\ \text { LUAD } & 0.98(0.95 \sim 1.01) & 0.13000 \\ \text { LUSC } & 1.06(1.01 \sim 1.11) & 0.02400 \\ \text { MESO } & 1.07(1.02 \sim 1.13) & 0.01200 \\ \text { OV } & 1.04(0.99 \sim 1.1) & 0.12000 \\ \text { PAAD } & 1.01(0.97 \sim 1.05) & 0.54000 \\ \text { PCPG } & 2.38(1.37 \sim 4.15) & 0.00220 \\ \text { PRAD } & 1.15(0.59 \sim 2.28) & 0.68000 \\ \text { READ } & 0.7(0.48 \sim 1.04) & 0.07900 \\ \text { SARC } & 0.99(0.93 \sim 1.05) & 0.72000 \\ \text { SKCM } & 0.95(0.91 \sim 0.99) & 0.02500 \\ \text { STAD } & 1.03(1 \sim 1.06) & 0.04900 \\ \text { TGCT } & 1.22(0.87 \sim 1.7) & 0.25000 \\ \text { THCA } & 0.95(0.65 \sim 1.38) & 0.78000 \\ \text { THYM } & 0.52(0.2 \sim 1.4) & 0.20000 \\ \text { UCEC } & 0.95(0.87 \sim 1.04) & 0.27000 \\ \text { UCS } & 0.96(0.8 \sim 1.15) & 0.64000 \\ \text { UVM } & 1.49(1.02 \sim 2.17) & 0.03800 \\ & & \end{array}$

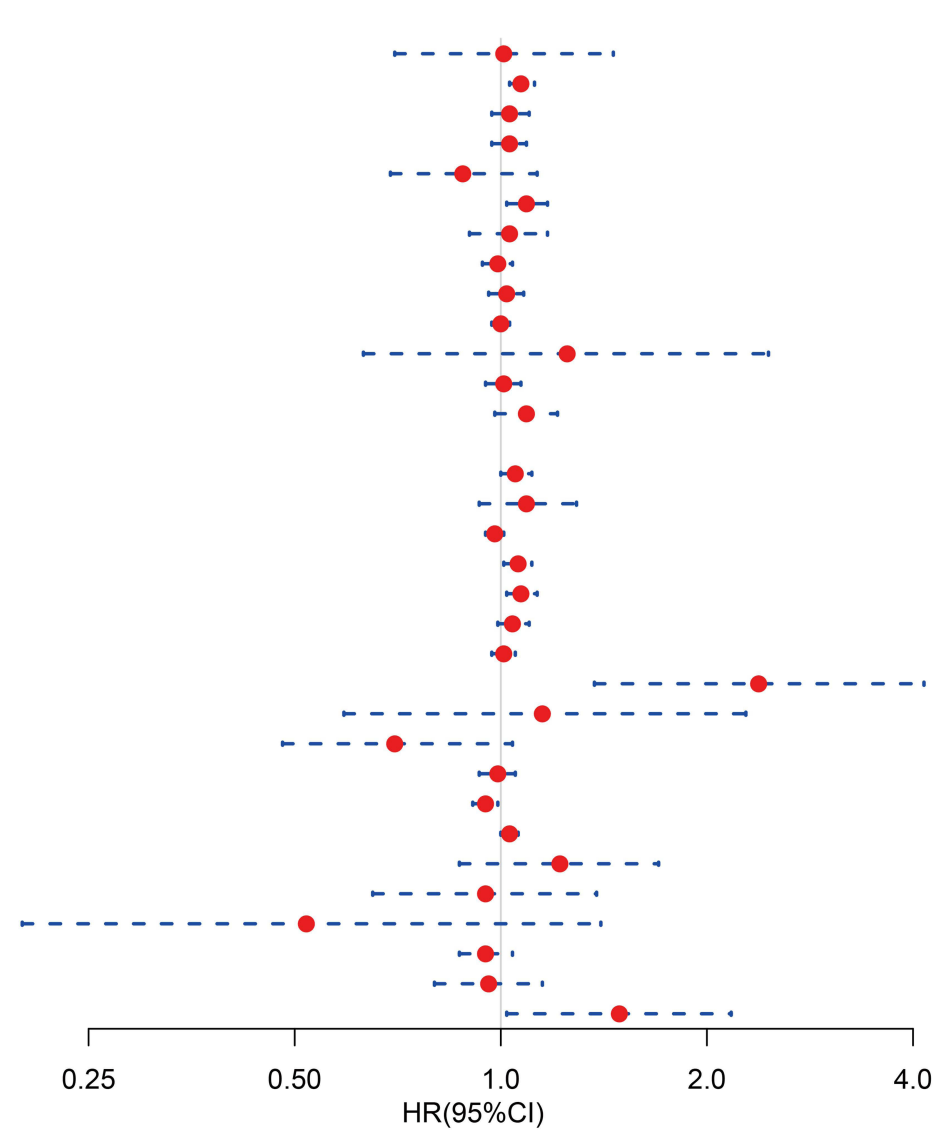

B
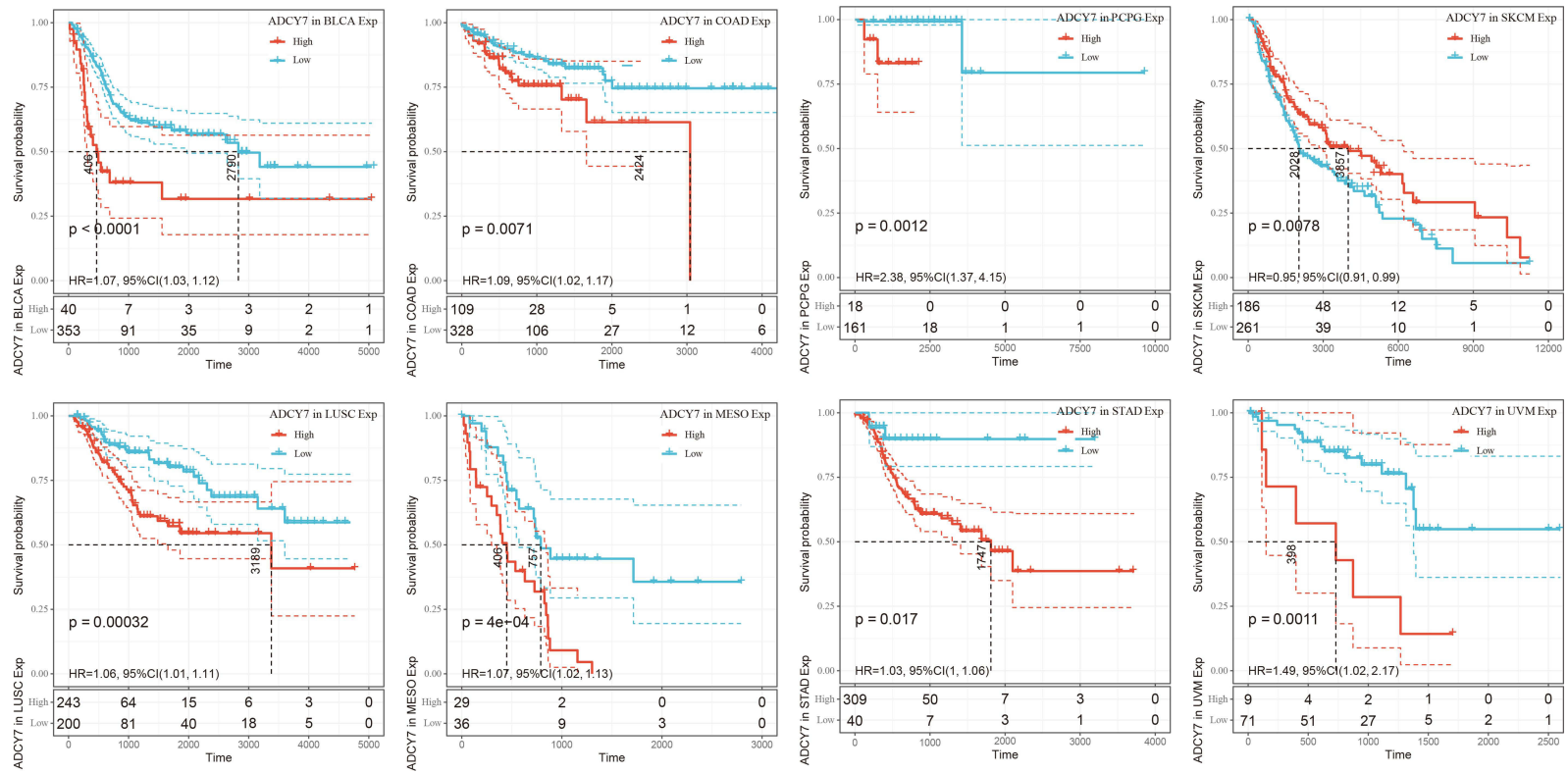

Figure 7 The relationship between ADCY7 expression and DSS. (A) Forest plots of hazard ratios of ADCY7 in thirty-three kinds of cancers. (B) KM curves showed that ADCY7 expression was correlated with DSS in 8 kinds of cancers (BLCA, COAD, PCPG, SKCM, UVM, STAD, MESO, and LUSC).

Abbreviations: ADCY7, Adenylate cyclase 7; DSS, Disease-specific survival. 


\begin{tabular}{|c|c|c|}
\hline $\mathrm{ACC}$ & $\begin{array}{r}\mathrm{HR} \\
114(0.89 \sim 147)\end{array}$ & $\begin{array}{l}\text { P Value } \\
0.29000\end{array}$ \\
\hline BLCA & $1.07(1.03 \sim 1.11)$ & 0.00026 \\
\hline BRCA & $1.01(0.97 \sim 1.06)$ & 0.60000 \\
\hline CESC & $1.02(0.97 \sim 1.08)$ & 0.39000 \\
\hline $\mathrm{CHOL}$ & $0.85(0.67 \sim 1.07)$ & 0.16000 \\
\hline COAD & $1.04(0.97 \sim 1.1)$ & 0.27000 \\
\hline DLBC & $1.03(0.95 \sim 1.1)$ & 0.51000 \\
\hline ESCA & $1(0.97 \sim 1.04)$ & 0.87000 \\
\hline GBM & $1.04(0.98 \sim 1.11)$ & 0.20000 \\
\hline HNSC & $1(0.97 \sim 1.02)$ & 0.74000 \\
\hline $\mathrm{KICH}$ & $1.07(0.58 \sim 1.96)$ & 0.83000 \\
\hline KIRC & $0.99(0.94 \sim 1.04)$ & 0.61000 \\
\hline KIRP & $1.02(0.93 \sim 1.12)$ & 0.65000 \\
\hline LAML & $\mathrm{NA}(\mathrm{NA} \sim \mathrm{NA})$ & \\
\hline LGG & $1.04(1 \sim 1.08)$ & 0.06200 \\
\hline LIHC & $1.05(0.92 \sim 1.2)$ & 0.50000 \\
\hline LUAD & $0.99(0.97 \sim 1.01)$ & 0.30000 \\
\hline LUSC & $1.05(1.01 \sim 1.09)$ & 0.00890 \\
\hline MESO & $1.05(1 \sim 1.1)$ & 0.07300 \\
\hline OV & $0.99(0.94 \sim 1.04)$ & 0.68000 \\
\hline PAAD & $1(0.97 \sim 1.04)$ & 0.84000 \\
\hline PCPG & $1.32(0.95 \sim 1.84)$ & 0.10000 \\
\hline PRAD & $1.15(0.99 \sim 1.35)$ & 0.07000 \\
\hline READ & $1.06(0.95 \sim 1.18)$ & 0.28000 \\
\hline SARC & $1(0.96 \sim 1.05)$ & 0.85000 \\
\hline SKCM & $0.98(0.95 \sim 1.01)$ & 0.23000 \\
\hline STAD & $1.03(1 \sim 1.06)$ & 0.06200 \\
\hline TGCT & $0.95(0.84 \sim 1.08)$ & 0.45000 \\
\hline THCA & $1.03(0.91 \sim 1.15)$ & 0.66000 \\
\hline THYM & $1.03(0.93 \sim 1.15)$ & 0.56000 \\
\hline UCEC & $0.99(0.94 \sim 1.04)$ & 0.65000 \\
\hline UCS & $0.96(0.81 \sim 1.14)$ & 0.64000 \\
\hline UVM & $1.81(1.26 \sim 2.59)$ & 0.0012 \\
\hline
\end{tabular}

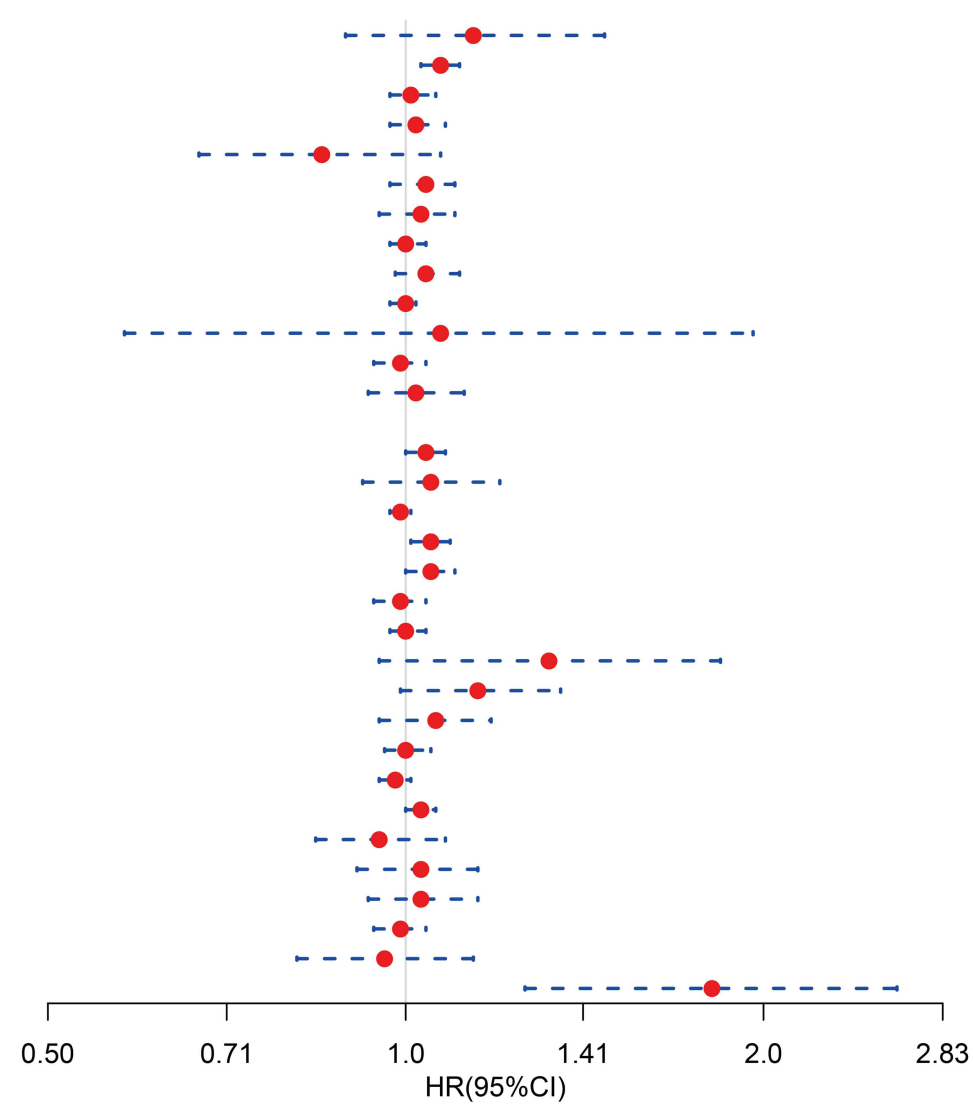

B
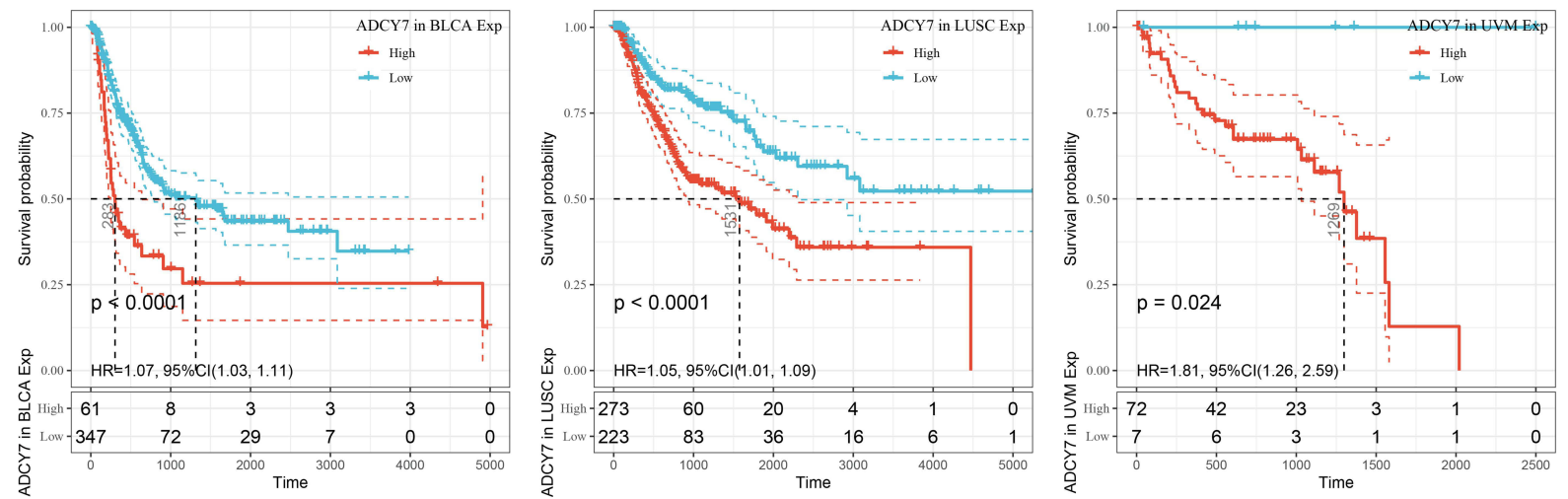

Figure 8 The relationship between ADCY7 expression and patients' PFI. (A) Forest plots of hazard ratios of ADCY7 in thirty-three kinds of cancers. (B) KM curves showed that ADCY7 expression was correlated with PFI in 3 kinds of cancers (BLCA, UVM, and LUSC).

Abbreviations: ADCY7, Adenylate cyclase 7; PFI, Progression-free interval.

dMMR/MSI-H solid tumor, immunotherapy can benefit regardless of the tissue type of tumor origin. ${ }^{34,35}$ Studies have shown that TMB levels can influence a patient's response to immune checkpoint inhibitors. ${ }^{36,37}$ MSI-H was also associated with increased TMB levels. ${ }^{38}$ Therefore, further investigation of the relationship between ADCY7 expression level and MMR gene expression, MSI, and TMB level in patients with tumors is necessary. In this study, the expression of ADCY7 was related to the expression of 5 MMR genes (MLH1, MSH2, MSH6, PMS2, and EPCAM) in most of the human cancers, with ACC, GBM as the exceptions. Moreover, ADCY7 expression was related to both MSI and TMB in COAD. More studies are needed to confirm whether ADCY7 can be used as a predictor of the efficacy of COAD immunotherapy.

Cancer immunotherapy has attracted the attention of many researchers. Studies have found that the expression 
G protein-coupled receptor signaling pathway, coupled to cyclic nucleotide second messenger adenylate cyclase-modulating $G$ protein-coupled receptor signaling pathway cyclic-nucleotide-mediated signaling second-messenger-mediated signaling cAMP-mediated signaling adenylate cyclase-activating $G$ protein-coupled receptor signaling pathway adenylate cyclase-inhibiting $G$ protein-coupled receptor signaling pathway cellular response to glucagon stimulus renal system process response to glucagon

cAMP-dependent protein kinase complex heterotrimeric G-protein complex GTPase complex ciliary base extrinsic component of cytoplasmic side of plasma membrane cytoplasmic side of plasma membrane extrinsic component of plasma membrane
cytoplasmic side of membrane integral component of postsynaptic membrane ciliary part

catecholamine binding $G$ protein-coupled receptor binding $\mathrm{G}$-protein beta/gamma-subunit complex binding $\mathrm{G}$ protein-coupled amine receptor activity adrenergic receptor activity peptide receptor activity peptide hormone binding peptide binding dopamine neurotransmitter receptor activity neurotransmitter receptor activity
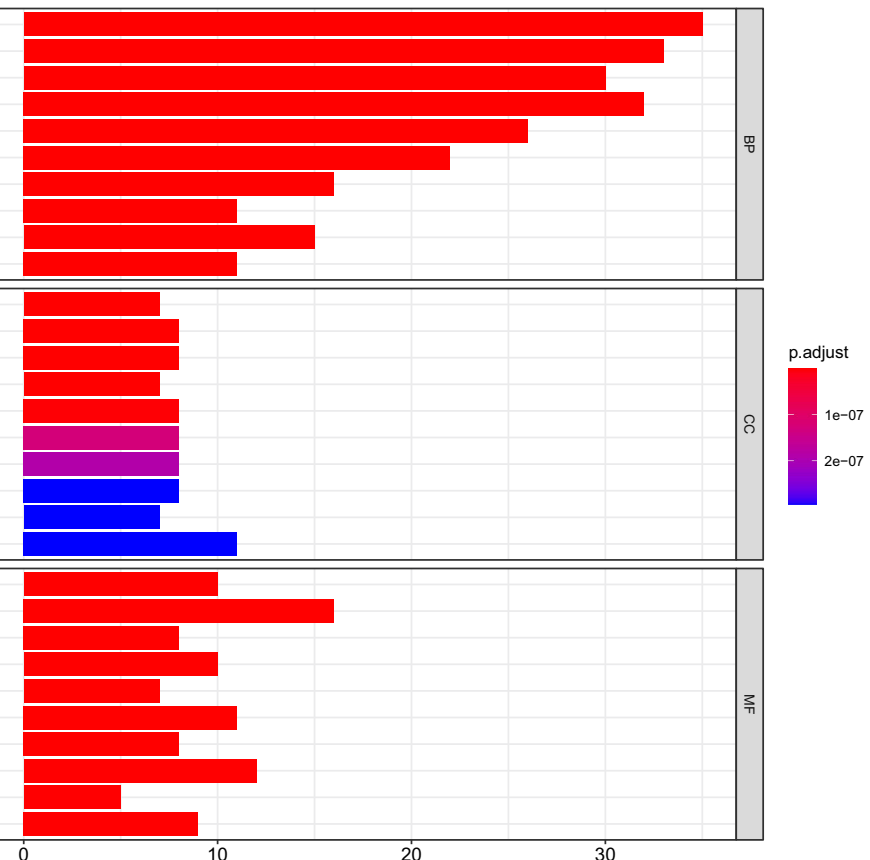

Figure $9 \mathrm{GO}$ analysis of ADCY7 and its correlated genes.

Abbreviations: ADCY7, Adenylate cyclase 7; GO, Gene Ontology.

Neuroactive ligand-receptor interaction cAMP signaling pathway Dopaminergic synapse Morphine addiction Renin secretion

Circadian entrainment Regulation of lipolysis in adipocytes Glutamatergic synapse Gap junction Serotonergic synapse

GABAergic synapseRelaxin signaling pathway

Cholinergic synapse Alcoholism

Cushing syndrome

Cocaine addiction cGMP-PKG signaling pathway Melanogenesis

Human cytomegalovirus infection Retrograde endocannabinoid signaling Adrenergic signaling in cardiomyocytes

Apelin signaling pathway

Estrogen signaling pathway-

Gastric acid secretion

Calcium signaling pathway

Cortisol synthesis and secretion Parathyroid hormone synthesis, secretion and action -

Chemokine signaling pathway

Oxytocin signaling pathway Growth hormone synthesis, secretion and action

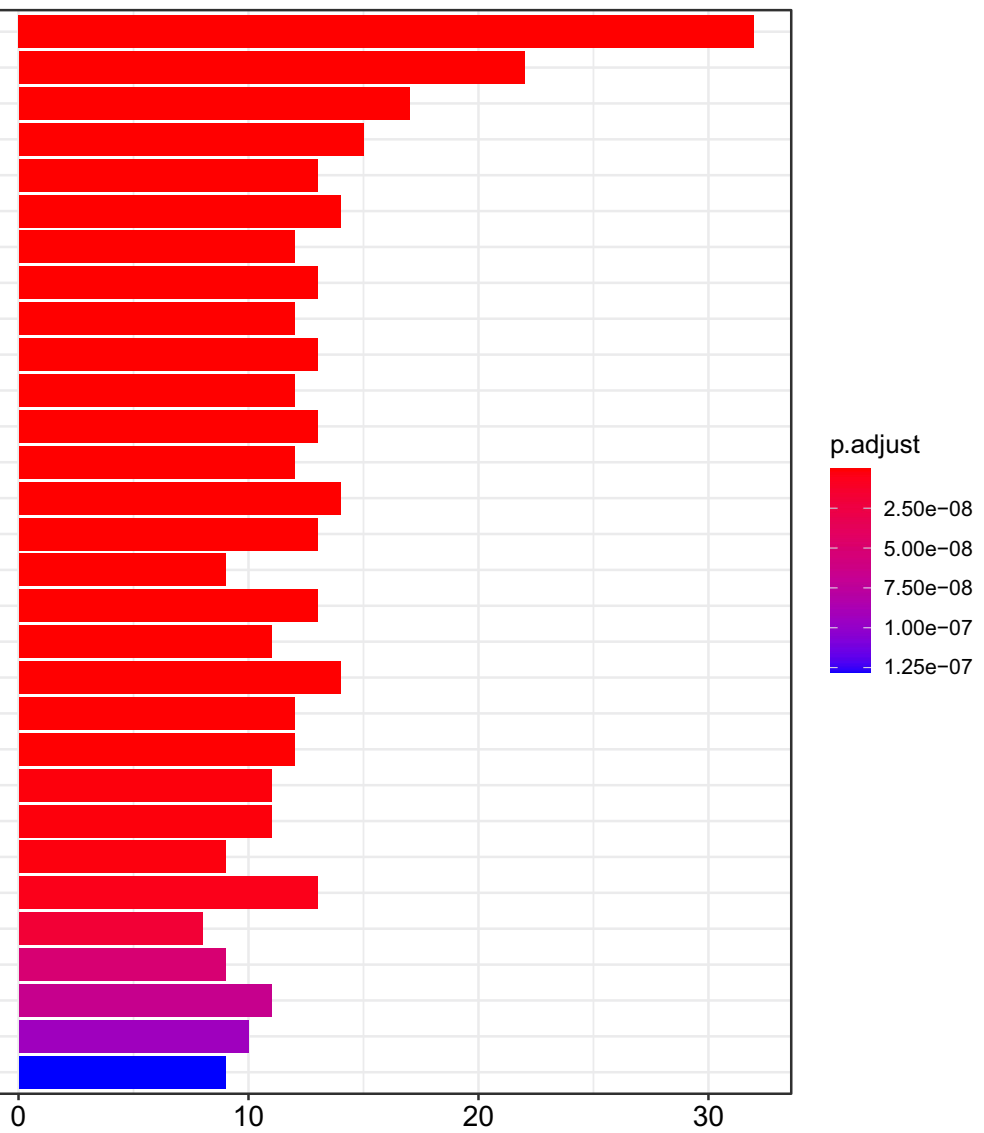

Figure 10 KEGG analysis of ADCY7 and its correlated genes.

Abbreviations: ADCY7, Adenylate cyclase 7; KEGG, Kyoto Encyclopedia of Genes and Genomes. 
level of ADCY7 is related to cancer immunity. Tumor immune microenvironment is mainly composed of tumor-infiltrating lymphocytes and other immune cells, which inhibit or penetrate tumor tension. ${ }^{39,40}$ Tumorinfiltrating lymphocytes are a new generation of antitumor adaptive immune effector cells that can be used as a prognostic marker of cancer. ${ }^{40}$ Immune escape is involved in the development of cancer. ${ }^{41}$ Macrophages are involved in anti-tumor immune response. ${ }^{42}$ These findings suggest that tumor-infiltrating immune cells are involved in tumor progression. In this study, ADCY7 expression was significantly related to six immuneinfiltrating cells in ACC, BRCA, and CHOL. Therefore, further investigation of the molecular mechanism of ADCY7 in tumor immunity is warranted.

This study also found that the expression of ADCY7 was related to poor prognosis in several types of tumors especially in BLCA and LUSC. Regardless of OS, PFI, or DSS analysis, the survival time of low ADCY7 expression in patients with BLCA and LUSC was longer than that in patients with BLCA and LUSC patients having high ADCY7 expression. This indicated that ADCY7 is a potential prognostic biomarker for BLCA and LUSC.

\section{Conclusion}

This study found that ADCY7 is a potential prognostic biomarker for many tumors, and the expression level of ADCY7 is related to cancer immunity. These results are based on online data, which means that further experiments are needed to investigate the mechanism of ADCY7 in cancer. This study has provided a new perspective on the role of ADCY7 in human cancers.

\section{Ethical Statement}

The authors are accountable for all aspects of the work in ensuring that questions related to the accuracy or integrity of any part of the work are appropriately investigated and resolved. The authors confirm that the data is available from the corresponding author upon reasonable request.

\section{Acknowledgments}

We would like to thank BulletEdits for English language editing. Yu Zeng and Nanhong Li are co-first authors for this study.

\section{Author Contributions}

All authors made a significant contribution to the work reported, whether that is in the conception, study design, execution, acquisition of data, analysis and interpretation, or in all these areas; took part in drafting, revising or critically reviewing the article; gave final approval of the version to be published; have agreed on the journal to which the article has been submitted; and agree to be accountable for all aspects of the work.

\section{Funding}

The work was supported by the Natural Science Foundation of Guangdong Province (2021A1515011373).

\section{Disclosure}

The authors have no conflicts of interest to declare.

\section{References}

1. Hanoune J, Defer N. Regulation and role of adenylyl cyclase isoforms. Annu Rev Pharmacol Toxicol. 2001;41:145-174. doi:10.1146/annurev.pharmtox.41.1.145

2. Baron L, Gombault A, Fanny M, et al. The NLRP3 inflammasome is activated by nanoparticles through ATP, ADP and adenosine. Cell Death Dis. 2015;6:e1629-e.

3. Ludwig M-G, Seuwen K. Characterization of the human adenylyl cyclase gene family: cDNA, gene structure, and tissue distribution of the nine isoforms. J Receptors Signal Transd. 2002;22:79-110. doi:10.1081/RRS-120014589

4. Li C, Xie J, Lu Z, et al. ADCY7 supports development of acute myeloid leukemia. Biochem Biophys Res Commun. 2015;465:47-52.

5. Price T, Brust TF. Adenylyl cyclase 7 and neuropsychiatric disorders: a new target for depression? Pharmacol Res. 2019;143:106-112.

6. Jiang LI, Sternweis PC, Wang JE. Zymosan activates protein kinase A via adenylyl cyclase VII to modulate innate immune responses during inflammation. Mol Immunol. 2013;54:14-22. doi:10.1016/j. molimm.2012.10.027

7. Masugi Y, Tanese K, Emoto K, et al. Overexpression of adenylate cyclase-associated protein 2 is a novel prognostic marker in malignant melanoma. Pathol Int. 2015;65:627-634. doi:10.1111/pin.12351

8. Chen SL, Hu F, Wang DW, et al. Prognosis and regulation of an adenylyl cyclase network in acute myeloid leukemia. Aging. 2020;12:11864-11877. doi:10.18632/aging. 103357

9. Sethna F, Feng W, Ding Q, et al. Enhanced expression of ADCY1 underlies aberrant neuronal signalling and behaviour in a syndromic autism model. Nat Commun. 2017;8:14359. doi:10.1038/ ncomms 14359

10. Bray F, Ferlay J, Soerjomataram I, et al. Global cancer statistics 2018: GLOBOCAN estimates of incidence and mortality worldwide for 36 cancers in 185 countries. CA Cancer J Clin. 2018;68:394-424.

11. Consortium G. The GTEx Consortium atlas of genetic regulatory effects across human tissues. Science. 2020;369:1318-1330.

12. Cerami E, Gao J, Dogrusoz U, et al. The cBio cancer genomics portal: an open platform for exploring multidimensional cancer genomics data. Cancer Discov. 2012;2:401-404. doi:10.1158/2159-8290. CD-12-0095

13. Csiszar A, Balasubramanian P, Tarantini S, et al. Chemically induced carcinogenesis in rodent models of aging: assessing organismal resilience to genotoxic stressors in geroscience research. Geroscience. 2019;41:209-227. doi:10.1007/s11357-019-00064-4

14. Kim S, Wyckoff J, Morris AT, et al. DNA methylation associated with healthy aging of elderly twins. Geroscience. 2018;40:469-484. doi:10.1007/s11357-018-0040-0 
15. Masser DR, Hadad N, Porter H, et al. Analysis of DNA modifications in aging research. Geroscience. 2018;40:11-29. doi:10.1007/s11357018-0005-3

16. Tiffen J, Gallagher SJ, Filipp F, et al. EZH2 cooperates with DNA methylation to downregulate key tumor suppressors and IFN gene signatures in melanoma. J Invest Dermatol. 2020;140(12):24422454.e5. doi:10.1016/j.jid.2020.02.042

17. Le DT, Durham JN, Smith KN, et al. Mismatch repair deficiency predicts response of solid tumors to PD-1 blockade. Science. 2017;357:409-413. doi:10.1126/science.aan6733

18. Asaoka Y, Ijichi H, Koike K. PD-1 blockade in tumors with mismatch-repair deficiency. $N$ Engl J Med. 2015;373:1979.

19. Hause RJ, Pritchard CC, Shendure J, et al. Classification and characterization of microsatellite instability across 18 cancer types. Nat Med. 2016;22:1342-1350. doi:10.1038/nm.4191

20. Li T, Fan J, Wang B, et al. TIMER: a web server for comprehensive analysis of tumor-infiltrating immune cells. Cancer Res. 2017;77: e108-e10. doi:10.1158/0008-5472.CAN-17-0307

21. Szklarczyk D, Gable AL, Lyon D, et al. STRING v11: protein-protein association networks with increased coverage, supporting functional discovery in genome-wide experimental datasets. Nucleic Acids Res. 2019;47:D607-d13. doi:10.1093/nar/gky1131

22. Yu G, Wang LG, Han Y, et al. clusterProfiler: an R package for comparing biological themes among gene clusters. Omics. 2012;16:284-287. doi:10.1089/omi.2011.0118

23. Liu S, Xie X, Lei H, et al. Identification of key circRNAs/lncRNAs/ miRNAs/mRNAs and pathways in preeclampsia using bioinformatics analysis. Med Sci Monit. 2019;25:1679-1693. doi:10.12659/ MSM.912801

24. Kammer GM. The adenylate cyclase-cAMP-protein kinase A pathway and regulation of the immune response. Immunol Today. 1988;9:222-229. doi:10.1016/0167-5699(88)91220-0

25. Castro A, Jerez MJ, Gil C, et al. Cyclic nucleotide phosphodiesterases and their role in immunomodulatory responses: advances in the development of specific phosphodiesterase inhibitors. Med Res Rev. 2005;25:229-244. doi:10.1002/med.20020

26. Patel TB, Du Z, Pierre S, et al. Molecular biological approaches to unravel adenylyl cyclase signaling and function. Gene. 2001;269:13-25. doi:10.1016/S0378-1119(01)00448-6

27. Baron L, Gombault A, Fanny M, et al. The NLRP3 inflammasome is activated by nanoparticles through ATP, ADP and adenosine. Cell Death Dis. 2015;6:e1629.

28. Murray F, Insel PA. Targeting cAMP in chronic lymphocytic leukemia: a pathway-dependent approach for the treatment of leukemia and lymphoma. Expert Opin Ther Targets. 2013;17:937-949. doi: $10.1517 / 14728222.2013 .798304$
29. Li C, Xie J, Lu Z, et al. ADCY7 supports development of acute myeloid leukemia. Biochem Biophys Res Commun. 2015;465:47-52. doi:10.1016/j.bbrc.2015.07.123

30. Lancet T. Moving toward precision medicine. Lancet. 2011;378:1678. doi:10.1016/S0140-6736(11)61725-X

31. Cao Z, Zhang S. An integrative and comparative study of pan-cancer transcriptomes reveals distinct cancer common and specific signatures. Sci Rep. 2016;6:33398. doi:10.1038/srep33398

32. Armaghany T, Wilson JD, Chu Q, et al. Genetic alterations in colorectal cancer. Gastrointest Cancer Res. 2012;5:19-27.

33. Baretti M, Le DT. DNA mismatch repair in cancer. Pharmacol Ther. 2018;189:45-62. doi:10.1016/j.pharmthera.2018.04.004

34. Li K, Luo H, Huang L, et al. Microsatellite instability: a review of what the oncologist should know. Cancer Cell Int. 2020;20:16. doi:10.1186/s12935-019-1091-8

35. Herbst RS, Baas P, Perez-Gracia JL, et al. Use of archival versus newly collected tumor samples for assessing PD-L1 expression and overall survival: an updated analysis of KEYNOTE-010 trial. Ann Oncol. 2019;30:281-289. doi:10.1093/annonc/mdy545

36. Wu HX, Wang ZX, Zhao Q, et al. Tumor mutational and indel burden: a systematic pan-cancer evaluation as prognostic biomarkers. Ann Transl Med. 2019;7:640. doi:10.21037/ atm.2019.10.116

37. Havel JJ, Chowell D, Chan TA. The evolving landscape of biomarkers for checkpoint inhibitor immunotherapy. Nat Rev Cancer. 2019;19:133-150.

38. Chalmers ZR, Connelly CF, Fabrizio D, et al. Analysis of 100,000 human cancer genomes reveals the landscape of tumor mutational burden. Genome Med. 2017;9:34. doi:10.1186/s13073-017-0424-2

39. Junttila MR, de Sauvage FJ. Influence of tumour micro-environment heterogeneity on therapeutic response. Nature. 2013;501:346-354. doi:10.1038/nature 12626

40. Woo JR, Liss MA, Muldong MT, et al. Tumor infiltrating B-cells are increased in prostate cancer tissue. J Transl Med. 2014;12:30. doi:10.1186/1479-5876-12-30

41. Ju Q, Li XM, Zhang H, et al. BRCA1-associated protein is a potential prognostic biomarker and is correlated with immune infiltration in liver hepatocellular carcinoma: a pan-cancer analysis. Front $\mathrm{Mol}$ Biosci. 2020;7:573619. doi:10.3389/fmolb.2020.573619

42. Shan X, Zhang C, Wang Z, et al. Prognostic value of a nine-gene signature in glioma patients based on tumor-associated macrophages expression profiling. Clin Immunol. 2020;216:108430. doi:10.1016/j. clim. 2020.108430
International Journal of General Medicine

\section{Publish your work in this journal}

The International Journal of General Medicine is an international, peer-reviewed open-access journal that focuses on general and internal medicine, pathogenesis, epidemiology, diagnosis, monitoring and treatment protocols. The journal is characterized by the rapid reporting of reviews, original research and clinical studies across all disease areas. The manuscript management system is completely online and includes a very quick and fair peer-review system, which is all easy to use. Visit http://www.dovepress.com/ testimonials.php to read real quotes from published authors. 